\title{
Conservation analysis and pathogenicity prediction of mutant genes of ectodysplasin a
}

\author{
Fangqi He ${ }^{1,2+}$, Hongfeng Wang ${ }^{1,2 \dagger}$, Xiaoyu Zhang ${ }^{1}$, Qingping Gao ${ }^{1 *} \mathbb{D}$, Feng Guo ${ }^{1 *}$ and Chang Chen ${ }^{2}$
}

\begin{abstract}
Background: Hypohidrotic ectodermal dysplasia (HED) is a common recessive X-linked hereditary disease that affects the development of ectoderm. Gene mutations of ectodysplasin A (EDA) play key roles in process of this disease. In our preliminary study, three unknown mutation sites (c.878 T > G, c.663-697del and c.587-615del) were detected from the pedigrees of HED.
\end{abstract}

Methods: Conservation analysis of the related homologous proteins in 3 unknown EDA gene mutation sites was conducted using the University of California Santa Cruz (UCSC) Genome Browser database. SIFT and PolyPhen-2, the online gene function prediction software, were utilized to predict the pathogenicity of point mutation of c.878 T > G.

Results: All three unknown mutation sites were located in the highly-conserved region of EDA and possessed strong amino acid conservation among different species. In addition, the results of the pathogenicity prediction of point mutation of c.878 T > G by SIFT $(P=0.00)$ and PolyPhen-2 $(S=0.997)$ demonstrated that the mutation site had considerable pathogenicity theoretically.

Conclusions: The EDA mutations of c.878 T > G, c.663-697del and c.587-615del may be responsible for the pathogenesis of HED in their pedigrees.

Keywords: Hypohidrotic ectodermal dysplasia, Ectodysplasin a gene, Gene mutation, Related homologous proteins, Conservation, Pathogenicity

\section{Background}

Hypohidrotic ectodermal dysplasia (HED), also known as congenital ectodermal dysplasia syndrome, is a group of common hereditary diseases that affect the development of ectoderm. It often affects the development of more than two ectoderm-derived organs. The prevalence is approximately $1 / 100000$ [1]. Clinical manifestations of HED mainly include hypotrichosis, loss or maldevelopment of sweat glands and nail dysplasia [2]. The main oral symptoms are alveolar bone dysplasia, craniofacial deformity and loss of intermaxillary distance [3, 4]. A majority of patients present with congenital tooth dysplasia, small incisors or canine teeth in cone shape and even full-mouth anodontia $[5,6]$.

\footnotetext{
* Correspondence: dentgao@163.com; dentguo@126.com

${ }^{\dagger}$ Fangqi He and Hongfeng Wang contributed equally to this work.

1 Department of Prosthodontics, Xiangya Hospital, Central South University,

Changsha 410008, Hunan, China

Full list of author information is available at the end of the article
}

Most HED cases have an X recessive inheritance [7, 8] and their pathogenic gene is the EDA gene [9]. Approximately $95 \%$ of the total HED patients are induced by gene mutations such as nonsense mutation, missense mutation and small fragment deletion [10]. Like most recessive X-linked hereditary diseases, the HED pedigree members are primarily male, whereas the female carriers merely present with mild clinical manifestations [11]. About $30 \%$ of the female carriers are asymptomatic [12].

In a preliminary study carried out by authors of the current study, peripheral blood of 4 unrelated HED pedigrees was collected. The peripheral blood of 100 healthy adults who don't have the family history of congenitally missing tooth was used for comparison. The 4 symptomatic patients were subjected to a thorough clinical examination. Four male probands had typical clinical manifestations which were in line with $\mathrm{X}$-linked recessive inheritance. The gene sequencing results demonstrated that the mutation

(c) The Author(s). 2018 Open Access This article is distributed under the terms of the Creative Commons Attribution 4.0 International License (http://creativecommons.org/licenses/by/4.0/), which permits unrestricted use, distribution, and 
was only found in the symptomatic patients and had patient to patient variability [13], including two point mutations (c.466C > T, c.878 T > G) and two deletion mutations (c.663-697del, C.587-615del). When compared with the reported gene mutation sites, point mutation $(\mathrm{c} .466 \mathrm{C}>\mathrm{T})$ in one pedigree was identified as a pathogenic mutation. The same is reported by Monreal 1988 [14]. The other three mutation sites (c.878 T > G, c.663-697del and c.587-615del) were new unidentified mutations.

SNP (single-nucleotide polymorphism) is the most common genome mutation. There are about a million SNP loci among humans. The SNP that is located in the gene coding region and can change the amino acid sequence leading to a missense mutation. With the improvement in disease diagnosis, there is a higher possibility of discovering new missense mutations [15]. In current times, researchers tend to use the different kinds of bioinformatics software to make the preliminary forecasting of the pathogenicity of the new mutation, recognize the related risks of the disease and offer the theoretical foundation using the cell model and the mouse disease model. In this study, conservation analysis of the related homologous proteins in 3 unknown EDA gene mutation sites was conducted by referring to the UCSC Genome Browser database. The online gene function prediction software SIFT and PolyPhen-2 were adopted to predict the pathogenicity of point mutation of c.878 T>. The aim of the study is to offer a reference for elucidating the pathogenesis of HED.

\section{Methods}

\section{Database selection}

Human Genome version 19 (hg19), the reference version of the human genome in the UCSC database, was used as the reference genome data for conservative analysis of the sequence alignment and related homologous protein of mutation sites of c.878 T > G, c.663-697del and c.587-615del in EDA gene among different species. These species included human, chimpanzee, gorilla, rhesus, mouse, rat, dolphin, horse, dog, chicken and zebrafish.

\section{Online gene function prediction}

SIFT and Polyphen-2 are the two most commonly used online gene function prediction software. Their parameters are the system default. The above were used to make the function prediction of the SNP and the point mutation.

The software SIFT(Sorting Intolerant From Tolerant)(http://sift.jcvi.org/), based on the prediction principle of protein sequence homology-based tool, predicts the influence of amino acid substitution. The predict outcomes of amino acid substitutions that are caused by nucleotide mutation is calculated as a standardized score whose range of variation ranges from 0 to 1 . There are four criteria for rating: tolerated low confidence, tolerated, deleterious low confidence and deleterious. When the score of SNP is less than 0.5 , it means the mutation is deleterious. Hence, the SNP has a great influence on protein function. Therefore, a lower SNP score implies increasing harm.

The software syphen2 (Phenotyping Version2)(http:// genetics.bwh.harvard.edu/pph2/), based on the prediction principle of protein structural homology-based method, adopts the machine learning algorithm of Naive Bayes to assess the stability influence caused by amino acid change of SNP on the folding, interaction and conformation of the protein. In the predict outcomes, the SNP is also assigned a score. The difference from SIFT is that, an increase in the SNP score implies increasing harm. There are four criteria for rating: unknown, benign, possibly damaging and probably damaging. When the score is larger than 0.9 , it means the mutation is probably damaging.

\section{Results}

\section{Gene detection in HED pedigrees}

In our preliminary investigation, 4 unrelated HED pedigrees were chosen for genetic testing and 3 unknown EDA mutations were detected. These included c.878 T > G, c.663-697del and c.587-615del. Drawing from previous experiments, conservation analysis of relevant homologous proteins in three unknown mutations of EDA gene was performed in reference to the UCSC Genome Browser database. The pedigrees of probands (c.878 $\mathrm{T}>\mathrm{G}$ ) belonged to a large clinical pedigree. The probands (III7), his nephew (IV1) and younger male cousin (III9) presented with identical manifestations. The results of gene detection showed that there were c.878 T>G missense mutation in the EDA gene in the results of the propositus, his nephew and younger male cousin. There is heterozygous mutation c. $878 \mathrm{~T}>\mathrm{G}$ in the EDA gene of carriers (I2,II9,III4). No mutation was found in normal subjects (II2,II3,II11,III6). The above is the genotype and phenotype co-segregation phenomenon. It can be speculated that this mutation is a pathogenic mutation from the gene level (Fig. 1).

\section{Conservation analysis of gene mutations of EDA gene}

The mutation sites of EDA gene including p.L293R (c.878 T > G), p.T221fsX6 (c.663-697del) and p.P196fs X33 (c.587-615del) possessed strong amino acid conservation among different species, prompting that these three newly-discovered mutation sites of EDA gene were probably located in the vital function region of the coding genes during the process of species evolution (Fig. 2, Fig. 3). These findings were consistent with the genetic testing results of three unknown mutation sites. 

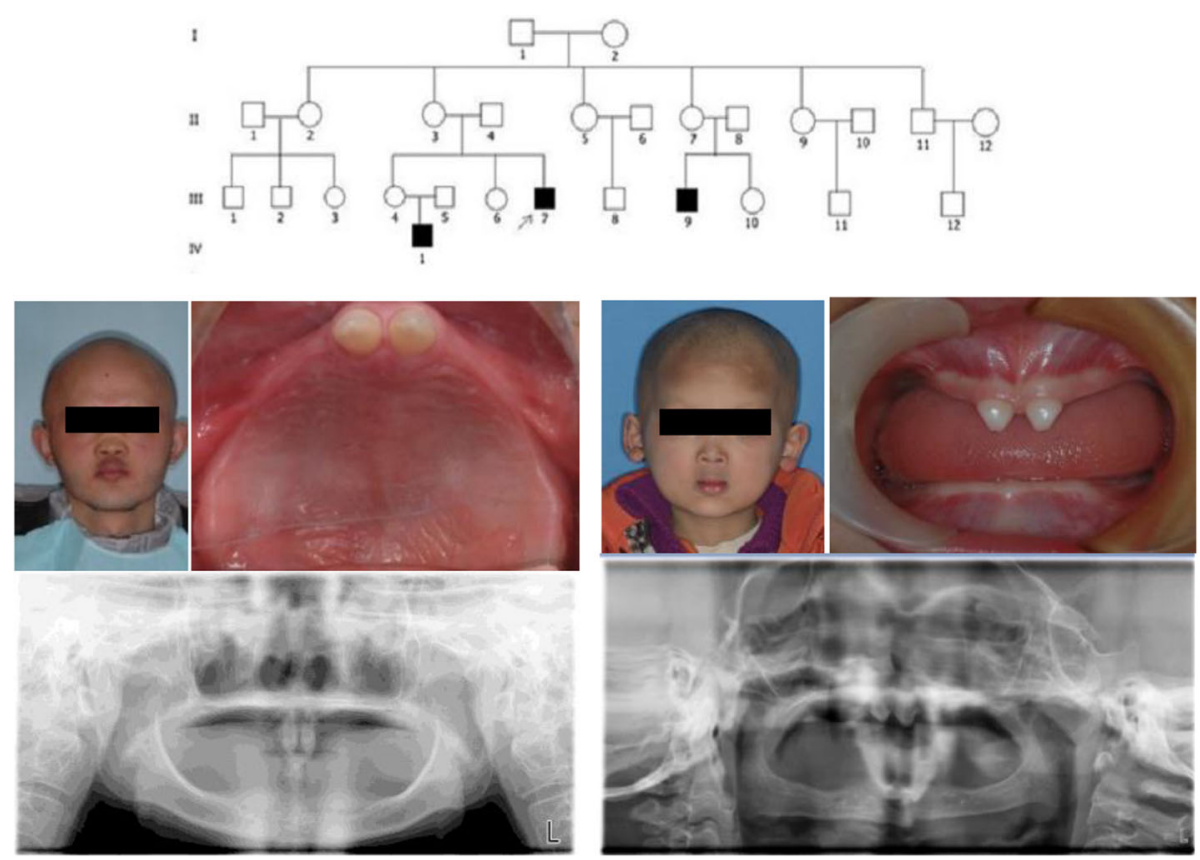

Fig. 1 The family tree of C.878T > G gene mutation patients affected by HED (upper) and their phenotypic appearance of the proband (lower left) and his nephew (lower right). Notes: HED, hypohidrotic ectodermal dysplasia

\section{Pathogenicity prediction of mutation site of c.878 T > G in EDA gene}

The pathogenicity of the mutation site of c.878 T > G was evaluated. SIFT revealed that the score of L293A was 0.00 and a score of $<0.05$ was considered to probably affect the protein function (Fig. 4). PolyPhen-2 demonstrated that the pathogenicity of mutation site of $c .878 \mathrm{~T}>\mathrm{G}$ was 0.997 , which was close to 1.0 , indicating that it theoretically had high pathogenicity and potentially caused damages (Fig. 5). The results above implied that this mutation site possessed strong pathogenicity theoretically.

\section{Discussion}

EDA gene is a causative gene of HED, which is located in the Xq12-q13 region. It contains 9 exons and encodes 391 amino acids. The encoded EDA protein is a type II trans-membrane protein with a short intracellular domain at the N-terminal and a TNF homology domain

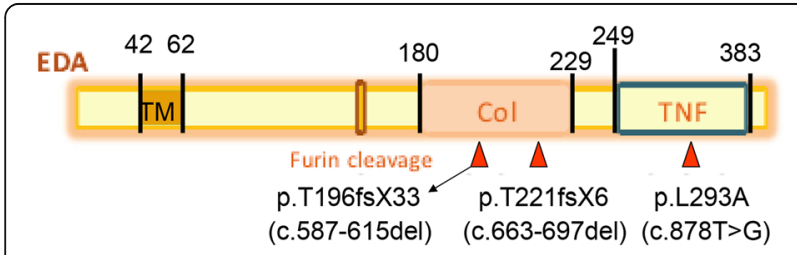

Fig. 2 The locations of three unknown EDA gene mutation sites in the functional domain of the EDA gene. Notes: EDA, ectodysplasin A; TM, transmembrane domain; Furin cleavage, furin enzyme binding site; Col, collagen-like domain TNF with 19 glycine residual repetitive sequence at the C-terminus [16]. It has multiple subtypes and mainly mediates epidermal-mesenchymal and cell-cell signaling transduction [17]. As an important member of tumor necrosis factor ligand family, EDA protein has been extensively studied in the field of developmental biology. It can regulate body morphogenesis and maintain the development of ectoderm-derived organs (tooth, hair, sweat gland and other cutaneous appendages) [18, 19]. Previous studies have demonstrated that application of the EDA gene-transfected bone mesenchymal stem cells can significantly improve the regenerative capacity of sweat glands and accelerate the potential repair and regeneration of the injured skin and its appendages [20]. However, after the EDA gene-inhibiting antibodies were implanted in wild-type pregnant mice, the wild-type mouse fetuses were found to have permanent ectodermal dysplasia [21].

EDA gene mutations are mainly located in four important functional areas [22]. (1) The junction (TM) between the trans-membrane and extra-cellular parts which is related to the polarity of the amino acids. (2) Furin cleavage is associated with the production of soluble molecules. The activity can be activated by the release of the soluble form of EDA proteins.(3) Collagen-like domain (collagen) can bind two or more EDA trimer together, thereby stimulating the downstream signaling pathway.(4) TNF homology domain (THD) can affect the formation of trimer and its specific binding with receptors. More than 
A
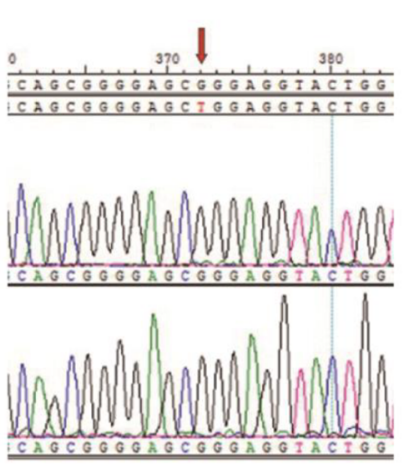

C
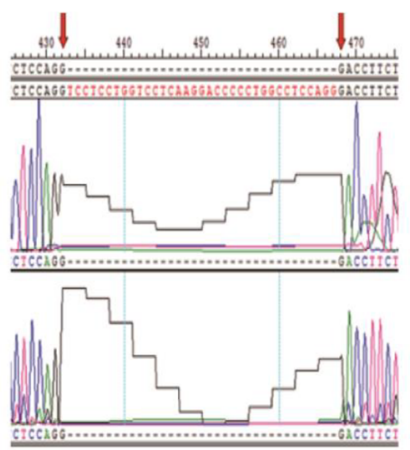

E
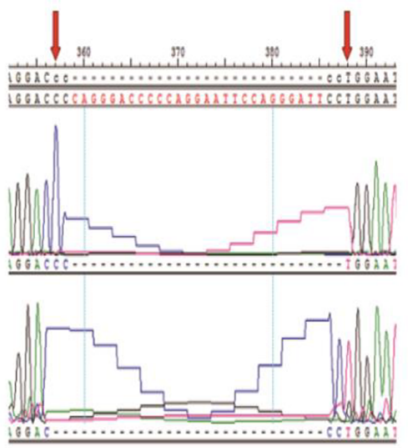

B

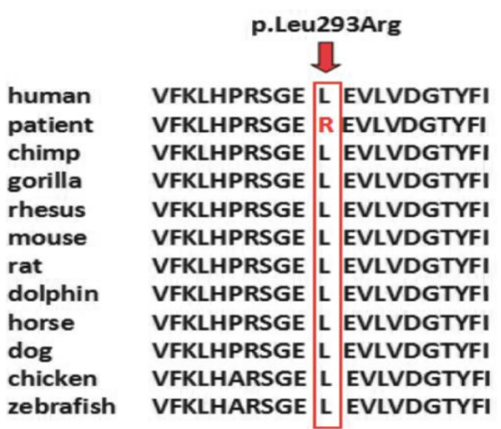

D

\begin{tabular}{lll|l}
\multicolumn{1}{c}{ p.T221fsX6 } \\
\begin{tabular}{ll|l} 
human \\
patient
\end{tabular} & PGPNGPPGPP & G & PPGPQGPPGI \\
chimp & PGPNGPPGPP & G & PPGPQGPPGI \\
gorilla & PGPNGPPGPP & G & PPGPQGPPGI \\
rhesus & PGPNGPPGPP & G & PPGPQGPPGI \\
mouse & PGPNGPPGPP & G & PPGPQGPPGI \\
rat & PGPNGPPGPP & G & PPGPQGPPGI \\
dolphin & PGPNGPPGPP & G & PPGPQGPPGI \\
horse & PGPNGPPGPP & G & PPGPQGPPGI \\
dog & PGPNGPPGPP & G & PPGPQGPPGI \\
chicken & PGPNGPQGPP & G & PPGPQGPPGI
\end{tabular}

F

\begin{tabular}{|c|c|c|}
\hline & p.P1 & 3 \\
\hline Ima & $\mathrm{Pr}$ & QGPPGIPGIPGIPGTTVMGPPGPPGPPGPQGPPGLQ \\
\hline tient & PPG & WNSRNNCYGTTWSSRSSWSSRTPWPPGTFWCC $X$ \\
\hline imp & PPG & P QGPPGIPGIPGIPGTTVMGPPGPPGPPGPQGPPGLQ \\
\hline rilla & PPG & QGPPGIPGIPGIPGTTVMGPPGPPGPPGPQGPPGLQ \\
\hline lesus & PPG & Q QGPPGIPGIPGIPGTTVMGPPGPPGPPGPQGPPGLQ \\
\hline ouse & PPG & Q QGPPGIPGIPGIPGTTVMGPPGPPGPPGPQGPPGLQ \\
\hline & PPG & P QGPPGIPGIPGIPGTTVMGPPGPPGPPGPQGPPGLQ \\
\hline & PPG & Q QGPPGIPGIPGIPGTTVMGPPGPPGPPGPQGPPGLQ \\
\hline 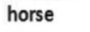 & PPG & P QGPPGIPGIPGIPGTTVMGPPGPPGPPGPQGPPGLQ \\
\hline & PPG & P QGPPGIPGIPGIPGTTVMGPPGPPGPPGPQGPPGLQ \\
\hline icken & PPC & P QGPPGIPGIPGIPGTTVMGPPGPPGPPGPQGPPGVQ \\
\hline rafish & PPC & P QGPPGIPGIPGIPGTTVMGPPGPPGPPGPQGPPGLQ \\
\hline
\end{tabular}

Fig. 3 Comparing the conservatism of three unknown EDA gene mutation sites among different species. (a) EDA gene mutation peak in the pedigree of c.878 T > G; (b) Conservation analysis of c.878 T > G mutation site among different species; (c) EDA gene mutation peak in the pedigree of c.663-697del; (d) Conservation analysis of c.663-697del mutation site among different species; (e) EDA gene mutation peak in the pedigree of c.587-615del; (f) Conservation analysis of c.587-615del mutation site among different species. These species included human, chimpanzee, gorilla, rhesus, mouse, rat, dolphin, horse, dog, chicken and zebrafish

100 types of EDA mutations have been found to have heterogeneity and ethnic differences [23, 24]. It is reported that $95 \%$ of the gene mutations occur in the $1,3,5,8$ and 9 exons, including missense mutation, small fragment insertion or deletion mutation and nonsense mutation [25, 26]. In addition, the deletion or translocation of single exon or full-length gene fragments has also been reported [27].

Mutations in the EDA gene can not only lead to congenital anodontia syndrome, but also provoke congenital anodontia with relatively mild symptoms, which are not complicated with the developmental defects of ectoderm-derived tissues and organs [26]. Tao et al. have reported of a Mongolian family with congenital anodontia. Besides congenital anodontia, no other clinical manifestations of HED were present in the affected family members. A new missense mutation (c.198C > G) was found in the EDA gene among all affected male and female carriers [28]. Song et al. [29] collected 15 unrelated male patients with asymptomatic congenital anodontia. Gene mutation testing revealed three novel mutation sites (p.Ala259Glu, p.Arg289Gys and p. 


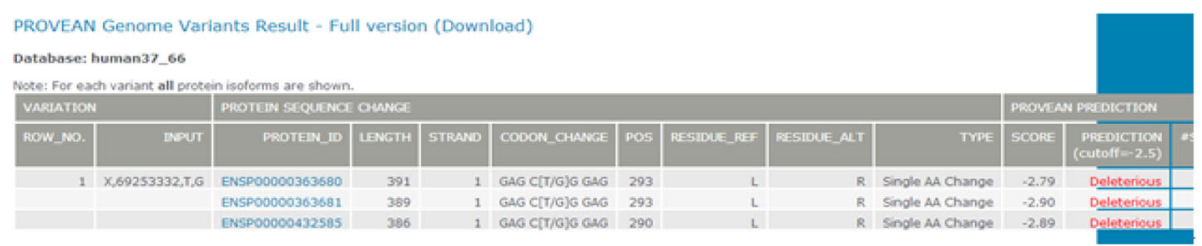

289R 0. 900.010 .000 .040 .060 .010 .010 .070 .010 .090 .010 .000 .060 .000 .061 .000 .030 .030 .010 .010 .00 290S 0. 870.030 .000 .140 .080 .000 .080 .010 .000 .030 .010 .000 .190 .010 .030 .031 .000 .050 .010 .010 .00 291G 0. 880.010 .000 .020 .000 .001 .000 .010 .000 .010 .000 .000 .040 .000 .000 .010 .000 .000 .000 .000 .02 292E 0. 930.030 .000 .021 .000 .010 .030 .040 .030 .130 .030 .010 .020 .010 .030 .040 .050 .050 .090 .000 .04 293L 0. 940.00.000.000.000.040.000.000.120.001.000.010.000.000.000.000.000.010.080.000.00 294E 0.960 .060 .000 .041 .000 .010 .040 .020 .080 .190 .070 .010 .040 .030 .070 .200 .060 .250 .100 .000 .01 295V 0. $960.010 .000 .000 .000 .000 .000 .000 .140,000.000 .000 .000 .000 .000,000.000 .001 .000,000.00$ 296L 0. 96 0.150.050.060.130. 200.100.220.130.151.000.060.140.110.120.130.120.130.140.070.29 $297 \mathrm{~V} 0.960 .360 .030 .360 .660 .020 .190 .060 .090 .480 .170 .050 .260 .130 .430 .310 .360 .301 .000 .010 .02$ 298D 0. $960,150,001.000 .090,000,030,000,010.040 .010,000,020.110,020,020.080 .120,010,000.00$ 299G 0. 960.000 .000 .000 .000 .001 .000 .000 .000 .000 .000 .000 .000 .000 .000 .000 .000 .000 .000 .000 .00 $300 \mathrm{~T} 0.960 .260 .090 .120 .140,430.170 .280 .260 .160 .540 .120 .160 .100 .150 .190 .211 .000 .320 .090 .60$ $\begin{array}{llllllllllllllllllllll}\text { pas } & \mathbf{A} & \mathbf{C} & \mathbf{D} & \mathbf{E} & \mathbf{F} & \mathbf{G} & \mathbf{H} & \mathbf{I} & \mathbf{K} & \mathbf{L} & \mathbf{I} & \mathbf{N} & \mathbf{P} & \mathbf{Q} & \mathbf{R} & \mathbf{S} & \mathbf{T} & \mathbf{V} & \mathrm{T} & \mathbf{Y}\end{array}$ 301 Y 0.960.000.000.000.000.030.000.000.000.000.000.000.000.000.000.000.000.000.000.001.00,

Fig. 4 The pathogenicity prediction of the mutation site of c.878 T > G using the software SIFT. The value of L293A was $P=0.00$, and a $P$ value $<$ 0.05 was considered to probably affect the protein function

Arg334His) in EDA gene of four patients with a detection rate of $27 \%$. The genetic defects of EDA gene could lead to the incidence of asymptomatic congenital anodontia.

Along with the development of genetics and genomics fields, the EDA genes originally found in both patients and mouse models have been proven to contain a highly-conserved TNF homology domain in vertebrates and mammals [22, 30]. The findings in this study have demonstrated that the c.878 $\mathrm{T}>\mathrm{G}$ mutation is located in the TNF homology domain of EDA gene. Mutations in this region can affect the binding of EDA protein to its receptors, resulting in the inactivation of NF- $\mathrm{kB}$ signaling pathway, thereby leading to the developmental defects of ectoderm-derived tissues and organs. NF- $\mathrm{KB}$ signaling pathway is now recognized as a classical signaling pathway associated with the incidence of HED [31]. C. $878 \mathrm{~T}>\mathrm{G}$ family is a large clinical pedigree. The nephews and cousins of the probands shared similar manifestations. Genetic testing showed that the base 878 of exon 7 was mutated from thymine to guanine in EDA gene of the probands and their nephews, and thus the CTG code was mutated into CGG, resulting in a change from leucine to arginine at the amino acid 293 (p.Leu293Arg). Genotype testing of this mutation at the gene level is the cause of hypoplastic ectodermal hypoplasia in this pedigree. In addition, further examination was carried out using the prediction software SIFT and PolyPhen-2. The results indicated that the mutation site possessed strong pathogenicity theoretically. The databases of 1000 Genome Project, the NHLBI GO Exome Sequencing Project and dbSNP database, were used to identify the frequency of the mutations. This further supported the pathogenicity of this mutation. However, the specific mechanism is not well understood. The study for the new missense mutation (c.878 $\mathrm{T}>\mathrm{G}$ ) aims to offer the theoretical support for the next functional experiment. Next, our research group will transfect the eukaryotic cell that is related to tooth development, and make a evaluate the effects of mutated EDA genes on

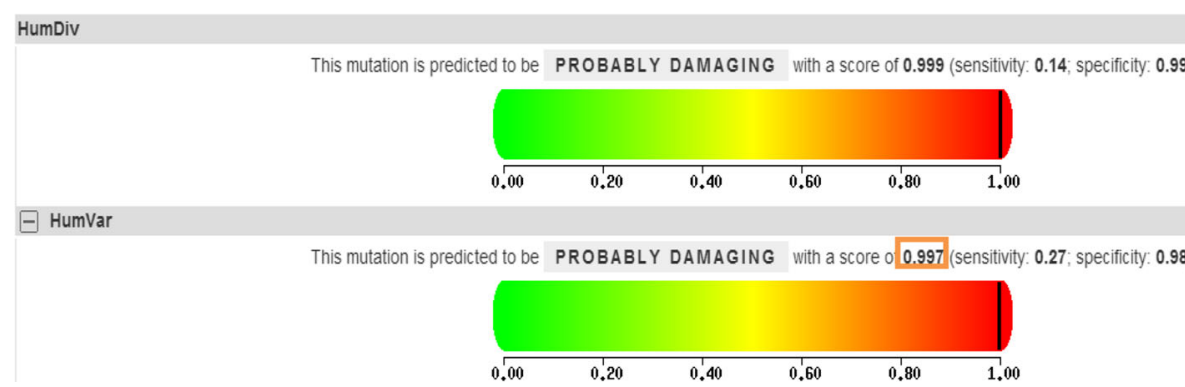

Fig. 5 The pathogenicity prediction of the mutation site of $c .878 \mathrm{~T}>\mathrm{G}$ using the software PolyPhen-2. The mutation prediction is based on the results of second graph HumVar. The closer the score is to 0.00 , the less possibility causing protein damage. The score of c.878 T > G was 0.997 , which was close to 1.0, indicating that it theoretically had high pathogenicity and potentially caused damages 
tissues and organs of ectodermal origin at the protein level with a goal of guiding the clinical treatment.

The other two mutations (c.663-697del, c.587-615del), which have not been reported, are located in the collagen-like domain of EDA gene. These mutations lead to the frame-shifting mutation of exon 4 and induce a termination codon in advance at the 226th and 238th amino acids, resulting in a truncated protein. This causes functional defects of the EDA protein, which is unable to form normal EDA trimer, weakens the activation of downstream signaling pathways and affects the development of ectoderm-derived tissues and organs. Due to the complexities involved in simulating computer algorithms, the numerical procedure to predict the functional effects of these two unknown deletion mutations was not used. This may be carried out further studies.

\section{Conclusions}

Three unknown mutation sites (c.878 T > G, c.663-697del and c.587-615del) from the pedigrees of HED possess strong conservation of amino acid among different species and the mutation site of c.878 T $>$ G holds strong pathogenicity theoretically. This may be responsible for the pathogenesis of HED in their pedigrees and it can also provide a reference for the pathogenicity of a wide range prediction of gene mutations.

\section{Abbreviations}

EDA: ectodysplasin A; HED: Hypohidrotic ectodermal dysplasia; UCSC: the University of California Santa Cruz

\section{Acknowledgements}

The authors would like to thank the medical editor from the United States providing language help, and Shouyi Lu for preliminary researchon this work

\section{Availability of data and material}

All data generated or analysed during this study are included in this published article.

\section{Funding}

The present study was supported by the Natural Science Foundation of Hunan Province (grant numbers 2017JJ2377); the Hunan Provincial Science and Technology Department of China (grant numbers 2015JC3038). The funding body conceived the experiments and provided data reference.

\section{Authors' contributions}

$\mathrm{FH}$ and HW performed the study, and were also major contributor in writing the manuscript. QG and FG conceived the experiments, and were the main proofreader of the manuscript. XZ and CC analyzed the data, and participated in the drafting of manuscript and the retrieval of database in later revision. All authors read and approved the final manuscript.

\section{Ethics approval and consent to participate}

The study was approved by the Ethical Committee of Xiangya Hospital in written (Number: 201703370), and written informed consent was obtained from the patients or their parents if the participants were minors.

\section{Consent for publication}

We have obtained written consent to publish from the participants, or their parents for minor participants, to report individual patient images and medical information.

\section{Competing interests}

The authors declare that they have no competing interests.

\section{Publisher's Note}

Springer Nature remains neutral with regard to jurisdictional claims in published maps and institutional affiliations.

\section{Author details}

${ }^{1}$ Department of Prosthodontics, Xiangya Hospital, Central South University, Changsha 410008, Hunan, China. ${ }^{2}$ Department of Prosthodontics, Changsha Stomatological Hospital, Changsha 410004, Hunan, China.

Received: 7 April 2018 Accepted: 27 November 2018

Published online: 07 December 2018

\section{References}

1. Winiewski SA, Kobielak A, Trzeciak WH, Kobielak K. Recent advances in understanding of the molecular basis of anhidrotic ectodermal dysplasia: discovery of a ligand, ectodysplasin a and its two receptors. J Appl Genet. 2002;43:97-107.

2. Visinoni AF, Lisboa T, Pagnan NA, Chautard EA. Ectodermal dysplasias: clinical and molecular review. Am J Med Genet A. 2009;149A:1980-2002.

3. Lesot $\mathrm{H}$, Clauss F, Manière MC, Schmittbuhl M. Consequences of X-linked hypohidrotic ectodermal dysplasia for the human jaw bone. Front Oral Biol. 2009:13:93-9.

4. Wu Y, Zhang C, Squarize CH, Zou D. Oral rehabilitation of adult edentulous siblings severely lacking alveolar bone due to ectodermal dysplasia: a report of 2 clinical cases and a literature review. J Oral Maxil Surg. 2015;73:1733.e1-12.

5. Itin PH, Fistarol SK. Ectodermal dysplasias. Am J Med Genet C Semin Med Genet. 2004;131C:45-51.

6. Ramesh K, Vinola D, John JB. Hypohidrotic ectodermal dysplasia-diagnostic aids and a report of 5 cases. J Indian Soc Pedod Prev Dent. 2010;28:47-54.

7. Zonana J, Elder ME, Schneider LC, et al. A novel X-linked disorder of immune deficiency and hypohidrotic ectodermal dysplasia is allelic to incontinentia pigmenti and due to mutations in IKK-gamma (NEMO). Am J Hum Genet. 2000:67:1555-62.

8. Cluzeau C, Hadj S, Jambou M, et al. Only four genes (EDA1, EDAR, EDARADD, and WNT10A) account for $90 \%$ of hypohidrotic/anhidrotic ectodermal dysplasia cases. Hum Mutat. 2011;32:70-2.

9. Kere J, Srivastava AK, Montonen O, et al. X-linked anhidrotic (hypohidrotic) ectodermal dysplasia is caused by mutation in a novel transmembrane protein. Nat Genet. 1996;13:409-16.

10. Deshmukh S, Prashanth S. Ectodermal dysplasia: a genetic review. Int J Clin Pediatr Dent. 2012;5:197-202.

11. Spfaer JA. A dental approach to carrier screening in X linked hypohidrotic ectodermal dysplasia. J Med Genet. 1981;18:459-60.

12. Liu Y, Yin W, Bian Z, Shi C. Causative gene analysis of Chinese Hypohidrotic ectodermal dysplasia pedigrees. J Oral Sci Res. 2015;31:41-4.

13. Lu SY, Gao QP, Zhang XY, He FQ, Chen RJ, Zeng TW, Yu HM. Mutation detection and analysis in EDA gene in four hypohidrotic ectodermal dysplasia families. International Journal of Stomatology. 2017;44:288-93.

14. Monreal AW, Zonana J, Ferguson B. Identification of a new splice form of the EDA1 gene permits detection of nearly all X-linked Hypohidrotic ectodermal dysplasia mutations. Am J Hum Genet. 1998:63:380-9.

15. Ng PC, Levy S, Huang J, et al. Genetic variation in an individual human exome[J]. PLoS Genet. 2008;4(8):e1000160.

16. Pummila $M$, Fliniaux I, Jaatinen $R$, James MJ, Laurikkala J, Schneider $P$, Thesleff I, Mikkola ML. Ectodysplasin has a dual role in ectodermal organogenesis:inhibition of bmp activity and induction of Shh expression. Development. 2007;134:117-25.

17. Monreal AW, Ferguson BM, Headon DJ, Street SL, Overbeek PA, Zonana J. Mutations in the human homologue of mouse dl cause autosomal recessive and dominant hypohidrotic ectodermal dysplasia. Nat Genet. 1999;22:366-9.

18. Blüschke G, Nüsken KD, Schneider H. Prevalence and prevention of severe complications of hypohidrotic ectodermal dysplasia in infancy. Early Hum Dev. 2010;86:397-9.

19. de AS, Paranaiba LM, Swerts MS, Martelli DR, de BL, Martelli JH. Orofacial features of hypohidrotic ectodermal dysplasia. Head Neck Pathol. 2012;6:460-6. 
20. Cai S, Pan Y, Han B, Sun TZ, Sheng ZY, Fu XB. Transplantation of human bone marrow-derived mesenchymal stem cells transfected with ectodysplasin for regeneration of sweat glands. Chin Med J (Engl) 201. 124: 2260-8.

21. Kowalczyk QC, Willen L, Dang AT, et al. Generation and characterization of function-blocking anti-ectodysplasin a (EDA) monoclonal antibodies that induce ectodermal dysplasia. J Biol Chem. 2014;289:4273-85.

22. Kowalczyk QC, Schneider P. Ectodysplasin a (EDA)-EDA receptor signalling and its pharmacological modulation. Cytokine Growth Factor Rev. 2014;25: 195-203.

23. Canueto J, Zafra CM, Ciria S, Unamuno P, González SR. A novel EDA gene mutation in a Spanish family with X-linked hypohidrotic ectodermal dysplasia. Actas Dermosifiliogr. 2011;102:722-5.

24. Conte C, Gambardella S, Bulli C, Rinaldi F, Di MD, Falconi M, Bramanti P, Desideri A, Novelli G. Screening of EDA1 gene in X-linked anhidrotic ectodermal dysplasia using DHPLC:identification of 14 novel mutations in Italian patients. Genet Test. 2008;12:437-42.

25. Tong DX, He SP, Wang LW, Jin L, Si P, Cheng X. Association of singlenucleotide polymorphisms in the cannabinoid receptor 2 gene with schizophrenia in the Han Chinese population. J Mol Neurosci. 2013;51:454-60.

26. Zhang J, Han D, Song SJ, Wang Y, Zhao H, Pan S, Bai B, Feng H. Correlation between the phenotypes and genotypes of $\mathrm{X}$-linked hypohidrotic ectodermal dysplasia and non-syndromic hypodontia caused by ectodysplasin-a mutations. Eur J Med Genet. 2011:54:377-82.

27. Orstavik KH, Knudsen GP, Nordgarden $H$, et al. Severe hypohidrotic ectodermal dysplasia in a girl caused by a de novo 9; X insertion that includes XIST and disrupts the EDA gene. Am J Med Genet A. 2007;143A: 1510-3.

28. Tao R, Jin B, Guo SZ, Qing W, Feng GY, Brooks DG, Liu L, Xu J, Li T, Yan Y, He L. A novel missense mutation of the EDA gene in a Mongolian family with congenital hypodontia. J Hum Genet. 2006;51:498-502.

29. Song S, Han D, Qu H, Gong Y, Wu H, Zhang X, Zhong N, Feng H. EDA gene mutations underlie non-syndromic oligodontia. J Dent Res. 2009;88:126-31.

30. Colosimo PF, Hosemann KE, Balabhadra S, et al. Widespread parallel evolution in sticklebacks by repeated fixation of Ectodysplasin alleles. Science. 2005;307:1928-33.

31. Zhang $Y$, Tomann $P$, Andl T, et al. Reciprocal requirements for EDA/EDAR/ NF-kappaB and Wnt/beta-catenin signaling pathways in hair follicle induction. Dev Cell. 2009;17:49-61.

Ready to submit your research? Choose BMC and benefit from:

- fast, convenient online submission

- thorough peer review by experienced researchers in your field

- rapid publication on acceptance

- support for research data, including large and complex data types

- gold Open Access which fosters wider collaboration and increased citations

- maximum visibility for your research: over $100 \mathrm{M}$ website views per year

At $\mathrm{BMC}$, research is always in progress.

Learn more biomedcentral.com/submissions 